\title{
ENVIRONMENT AND CLASSICAL CHANNELS IN CATEGORICAL QUANTUM MECHANICS
}

\author{
BOB COECKE $^{a}$ AND SIMON PERDRIX $^{b}$ \\ ${ }^{a}$ University of Oxford, Department of Computer Science, Quantum Group \\ e-mail address: coecke@cs.ox.ac.uk \\ ${ }^{b}$ CNRS, Laboratoire d'Informatique de Grenoble \\ e-mail address: simon.perdrix@imag.fr
}

\begin{abstract}
We present a both simple and comprehensive graphical calculus for quantum computing. In particular, we axiomatize the notion of an environment, which together with the earlier introduced axiomatic notion of classical structure enables us to define classical channels, quantum measurements and classical control. If we moreover adjoin the earlier introduced axiomatic notion of complementarity, we obtain sufficient structural power for constructive representation and elegant correctness derivation of many quantum informatic protocols, including classically controlled quantum teleportation and dense coding, and quantum key distribution.
\end{abstract}

\section{INTRODUCTION}

Categorical quantum mechanics Abramsky and Coecke 04 provides a new perspective on quantum information processing. Particularly appealing is the fact that the symmetric monoidal categorical language comes with an intuitive graphical calculus [Penrose 71, Joyal and Street 91. This approach has meanwhile led to new results in quantum information and quantum foundations.

Graph states, a key resource for the measurement based quantum computational model, and translations thereof to the circuit model were studied in Duncan and Perdrix 09, Duncan and Perdrix 10. There is a compositional framework for studying the structure of general multipartite quantum entanglement Coecke and Kissinger 10. Quantum theory as well as Spekkens' toy theories have been casted within a single mathematical framework, enabling corresponding analysis of quantum non-locality in terms of 'phase groups' Coecke, Edwards and Spekkens 11. There exists a no-cloning theorem for a very general class of theories Abramsky 09].

Meanwhile there also exists a software tool with not only a graphical interface but also a 'graphical internal logic', quantomatic, which (semi-)automates graphical reasoning. It has been developed in a collaboration between Oxford, Edinburgh and since recently Google, and involved interpreting theories formulated in symmetric monoidal language in

1998 ACM Subject Classification: F.1.1, F.3.2.

Key words and phrases: Categorical semantics, quantum computing, classical data.

DOI:10.2168/LMCS-8(4:14)2012

C) B. Coecke and S. Perdrix

(c) Creative Commons 
terms of 'open graphs' Dixon and Kissinger 11. An important fragment of the graphical language admits a completeness theorem with respect to Hilbert spaces Selinger 11b.

Categorical quantum mechanics notions relevant for this paper are:

(A) by endowing compact categories Kelly and Laplaza 80 with an identity-on-objects 'dagger'-involution, one can abstractly capture bipartite states, map-state duality, braket duality and unitary operations Abramsky and Coecke 04;

(B) one can assign to any dagger compact category $\mathbf{C}$ of pure states and operations another dagger compact category $C P M(\mathbf{C})$ of 'mixed states' and 'completely positive maps' Selinger 07; $C P M(\mathbf{C})$ can be axiomatized as a dagger compact category with for every object $A$ a privileged 'maximally mixed state' $\perp_{A}: \mathrm{I} \rightarrow A$ [Coecke 08];

(C) 'classical structures' Coecke and Pavlovic 07, Coecke, Pavlovic and Vicary 12, which are certain kinds of commutative Frobenius algebras [Carboni and Walters 87], enable one to handle classical data and control Coecke, Paquette and Pavlovic 09;

(D) from an axiomatization of 'complementarity' (or 'unbiasedness') basic quantum logic gates can be constructed [Coecke and Duncan 08, Coecke and Duncan 11].

The interaction of these concepts has not been subjected to a detailed study yet. Here, we distill the notion of environment out of (B), and by blending it in an appropriate manner with (C) we define the notion of classical channel; these interact in a particularly nice manner with (D) and together they provide a simple and elegant graphical calculus to represent and prove correctness of typical quantum computational protocols e.g. teleportation (including classical control) Bennett et al. 93, dense coding Bennett and Wiesner 92, and QKD protocols Bennett and Brassard 84, Ekert 91.

Our main point is to show how with very little structural effort one straightforwardly reproduces these non-trivial quantum behaviors. Furthermore, the simple explicit account that we obtain here on the classical-quantum interaction, which substantially simplifies the earlier work in this direction in Coecke, Paquette and Pavlovic 09], will enable a fully comprehensive purely diagrammatic study of quantum informatic situations involving complex information flows between the classical and the quantum. It moreover provides novel foundational insights on the nature of this interaction.

Section 2 outlines how we conceive the classical-quantum distinction. Section 3 recalls the notion of classical structure, and Section 4 discusses complementary (or unbiasedness) thereof. Section 5 introduces the notion of environment, and Section 6 combines environment and classical structure to form a classical channel, measurements (\$6.2) and control operations ( $\$ 6.1$ ), and studies the role of complementarity $-\$ 6.3$ provides an explicit interpretation of the graphical language within Hilbert space quantum mechanics for the specific case of qubits. In Section 7 we derive basic quantum informatic protocols. While we restrict ourselves to pure states and operations, our graphical framework straightforwardly extends to mixed states and operations, as indicated in Section 8 .

We assume that the reader is familiar with the basic concepts of quantum computation, such as states, operations, measurement and control. The graphical language for symmetric monoidal categories is surveyed in Selinger 11a], and a tutorial tailored towards applications in categorical quantum mechanics is Coecke and Paquette 11]. In this context, chapters $\S 2$ and $\S 4$ of Coecke and Duncan 11] are also useful. 


\section{Classicality vs. Quantumness}

Let $\mathcal{H}$ be a Hilbert space. By a cloning map one means an operation

$$
U: \mathcal{H} \otimes \mathcal{H} \rightarrow \mathcal{H} \otimes \mathcal{H}
$$

which is such that for all $|\psi\rangle \in \mathcal{H}$ and some $|0\rangle \in \mathcal{H}$ we have

$$
U(|\psi\rangle \otimes|0\rangle)=|\psi\rangle \otimes|\psi\rangle \text {. }
$$

When fixing $|0\rangle$ within the argument we can instead consider

$$
\Delta:=U(-\otimes|0\rangle): \mathcal{H} \rightarrow \mathcal{H} \otimes \mathcal{H}
$$

rather than $U$. It is well-known that there exists no cloning map, by the so-called no-cloning theorem Dieks 82, Wootters and Zurek 82. Now, if an operation clones certain pure states, say the basis vectors $\{|i\rangle\}_{i}$, this does not imply that it clones mixtures of these too; setting $\rho=\sum_{i} \rho_{i}|i\rangle\langle i|$ we have:

$$
\Delta \circ \rho \circ \Delta=\Delta \circ\left(\sum_{i} \rho_{i}|i\rangle\langle i|\right) \circ \Delta=\sum_{i} \rho_{i} \Delta \circ(|i\rangle\langle i|) \circ \Delta=\sum_{i} \rho_{i}|i i\rangle\langle i i| \neq \rho \otimes \rho .
$$

However, what we do have is that

$$
\operatorname{tr}_{1}(\Delta \circ \rho \circ \Delta)=\operatorname{tr}_{2}(\Delta \circ \rho \circ \Delta)=\rho,
$$

where $t r_{1}$ and $t r_{2}$ respectively trace out the first and the second system. For arbitrary completely positive maps $\mathcal{E}$ Eqs. (2.1) becomes:

$$
\operatorname{tr}_{1}(\mathcal{E}(\rho))=\operatorname{tr}_{2}(\mathcal{E}(\rho))=\rho .
$$

Universal validity of Eqs. (2.2) for a particular completely positive map $\mathcal{E}$ acting on the entire space of all density operators has been referred to as broadcasting. But the nobroadcasting theorem [Barnum et al. 96] states that only mixed states which share a basis in which they all are diagonal (i.e. mixed states that can be jointly simulated by classical probability distributions) can be broadcast by the same completely positive map.

From the above discussion it follows that broadcastability is a strictly weaker requirement than cloneability. We have:

\begin{tabular}{c|c|c|c|c|} 
& pure classical & mixed classical & pure quantum & mixed quantum \\
\hline broadcastable: & YES & $\underline{\text { YES }}$ & NO & NO \\
\hline cloneable: & yes & $\underline{\text { no }}$ & no & no \\
\hline
\end{tabular}

where by classical we refer to a set of density operators that are diagonal in the same basis. So (not non-cloneability but) non-broadcastability 'identifies' quantum relative to classical, in that classical states, both pure and mixed, can always be broadcast by a single quantum operation, while this is not possible for quantum states that cannot be jointly simulated classically. Now, taking the contrapositive, for us classicality will mean broadcastability.

Equivalently, one can also conceive classicality as the result of decoherence [Zurek 91. Concretely, 'total' decoherence is the completely positive map which erases all non-diagonal elements in the matrix representation of a given basis, that is,

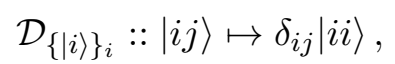

where $\delta_{i j}$ is the Kronecker delta. Broadcasting and decoherence are indeed closely related: in the case of the first 'one copies into the environment', while in the case of the second 'one couples to the environment'. Decoherent then means invariance under this coupling. Formally, decoherent density operators relative to a fixed $\mathcal{D}_{\{|i\rangle\}_{i}}$ are exactly those collections 
of density operators that can be jointly broadcast; they all are diagonal in the basis $\{|i\rangle\}_{i}$ and hence can be simulated by the classical probability distributions that make up the diagonals.

Summarizing the above:

classical $:=$ broadcastable $\equiv$ decoherent

We will treat 'classicality' as a 'behavior' -i.e. behaves as if it is classical in the above discussed sense- rather than as the specification of the actual physical realization of a system. An important point in this context, already realized in Coecke and Pavlovic 07, Coecke, Paquette and Pavlovic 09, is that by taking quantum to be the 'default behavior' within the mathematical universe of all operations, characterization of classical entities can be done in purely diagrammatic terms. In the concrete Hilbert space realization, this means that one only needs to rely on the multiplicative tensor product structure as a primitive connective, with no reference to the additive vector space structure. One could refer to this as 'classicization', in contrast to the standard notion of 'quantization' where one starts with a classical theory and then freely adjoins the additive vector space structure.

As an example, consider a quantum measurement, which when applied to a quantum system changes the state of that quantum system and produces classical data. Since the resulting quantum state is an eigenstate for that measurement, hence broadcastable, it behaves precisely in the same manner as the classical data does. As a result, in the graphical calculus the classical data and the collapsed state won't be distinguishable once we omit explicit specification wether physically they are either classical or quantum. This ambiguity captures a feature of the particular manner in which quantum and classical data interact, namely, that the creation of classical data renders the quantum state in an eigenstate. Of course, if we later apply a non-classical unitary to the resulting quantum state, then we reassert its proper quantumness.

Put in type-theoretic terms, there will be no such thing as a fixed 'classical type' and fixed 'quantum type' in our representation, since we can abstract away over these 'implementation details' without altering the essential structure. Of course, one can add those details in order to connect the graphical language to concrete physical protocols where the classical quantum distinction may be fundamental for the conceptual analysis, for example, in quantum teleportation it is crucial that the classical communication can indeed be realized by purely classical finitary means. In Section 7 we give several examples of protocols that come with specification of what is classical and what is quantum, and then pass to the abstract diagrammatic calculus where forgetting the physical realization is essential to perform the computation.

\section{Classical structures}

In this paper we will work in the graphical representation of symmetric monoidal categories JJoyal and Street 91. Mac Lane's strictification theorem [Mac Lane 00, p.257] allows us to take our symmetric monoidal categories to be strict, that is:

$$
(A \otimes B) \otimes C=A \otimes(B \otimes C) \quad \text { and } \quad A \otimes \mathrm{I}=A=\mathrm{I} \otimes A .
$$

Morphisms $f: A_{1} \ldots A_{n} \rightarrow B_{1} \ldots B_{m}$, which we interpret as processes are respectively represented as boxes where the input wires represent the objects $A_{1} \ldots A_{n}$ and the output 
wires represent the objects $B_{1} \ldots B_{m}$ :

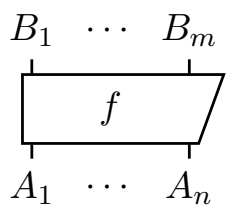

Other shapes may be used to emphasize extra structure. Elements $s: \mathrm{I} \rightarrow A_{1} \ldots A_{n}$, which in the graphical representation have no inputs, are interpret as 'states', and co-elements $e: B_{1} \ldots B_{m} \rightarrow$ I with no outputs are interpreted as 'effects'. In standard quantum notation they would be kets $|\psi\rangle$ and bras $\langle\psi|$ respectively. Composition and tensoring are respectively represented as:

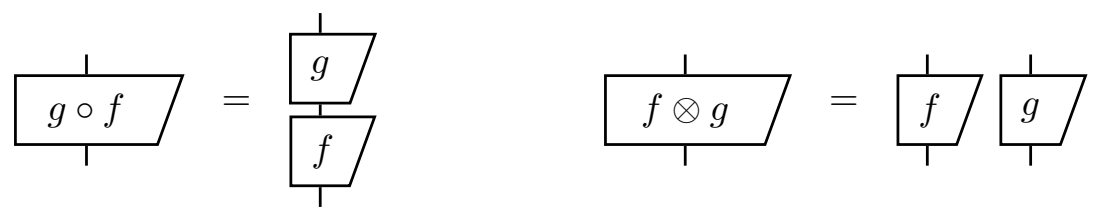

Theorem 3.1. Joyal and Street 91, Selinger 11a An equation follows from the axioms of symmetric monoidal categories if and only if it can be derived in the graphical language via diagram isomorphisms.

A dagger functor on a symmetric monoidal category [Selinger 07] is an identity-onobjects contravariant involutive strict monoidal functor. It is graphically represented by flipping pictures upside-down, for instance:

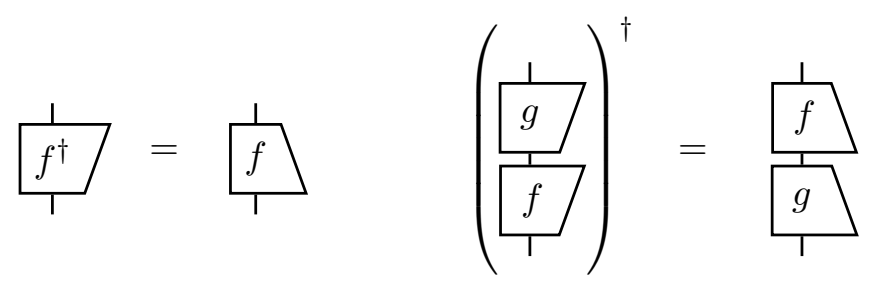

Given such a dagger functor, a morphism $f: A \rightarrow B$ is an isometry if $f^{\dagger} \circ f=1_{A}$, and it is unitary of both $f$ and $f^{\dagger}$ are isometries.

A dagger compact category Abramsky and Coecke 05 is a dagger symmetric monoidal category in which each object $A$ comes with two morphisms $\eta_{A}: \mathrm{I} \rightarrow A^{*} \otimes A$ and $\epsilon_{A}$ : $A \otimes A^{*} \rightarrow$ I which satisfy certain equations. In this paper we take all our objects to be self-dual, that is, $A=A^{*} 1$ Graphically, we represent $\eta_{A}$ as:

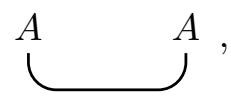

we take $\epsilon_{A}$ to be its dagger, and the equations that govern $\eta_{A}$ and $\epsilon_{A}$ are:
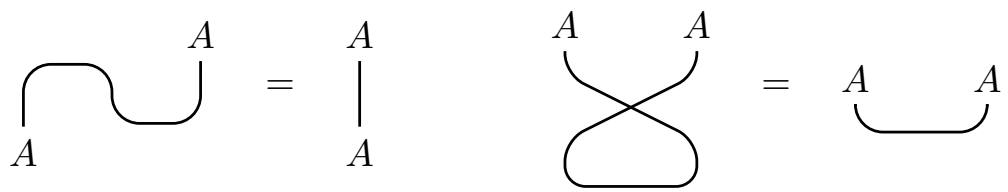

\footnotetext{
${ }^{1} \mathrm{~A}$ detailed study of the coherences for this situation is in Selinger 10.
} 
Remark 3.2. The results in this paper can be extended to the case of non-self-dual compact structures, by relying on the results in Coecke, Paquette and Perdrix 08. This would, for example, be required when considering all three complementary measurements on a qubit.

Theorem 3.3. Kelly and Laplaza 80, Selinger 07 An equation follows from the axioms of (dagger) compact categories if and only if it can be derived in the corresponding graphical language via isotopy.

The key difference between diagram isomorphism as in Theorem 3.1 and isotopy as in Theorem 3.3 is that diagram isomorphisms take specification of the boxes' inputs and outputs into account, while isotopy abstracts away these roles. Hence within the scope of Theorem 3.3 only the topology of the diagrams matters.

By classical structures [Coecke and Pavlovic 07] we mean internal commutative special dagger Frobenius algebras in a dagger compact category for which we also require 'compatibility with the compact structure' (see below). We won't give an explicit definition here, but will rely on a remarkable normal form result that holds for morphisms build from this structure, namely, any morphism

$$
\Xi_{n}^{m}: \underbrace{A \otimes \ldots \otimes A}_{n} \rightarrow \underbrace{A \otimes \ldots \otimes A}_{m}
$$

obtained by composing and tensoring the structural morphisms of a classical structure and the symmetric monoidal structure, and of which the diagrammatic representation is connected, only depends on $n$ and $m$ [Lack 04]. Graphically we represent this unique morphism as an $n+m$-legged spider:

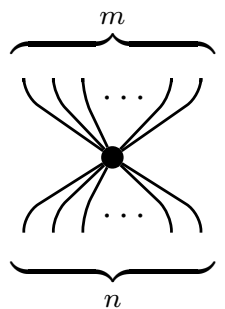

From the axioms of classical structures it follows that these spiders are invariant when one exchanges the roles of front-legs and back-legs, when one swaps two legs of either of these, and that the $(1+1)$-legged spider is the identity, that is,

$$
\left(\Xi_{n}^{m}\right)^{\dagger}=\Xi_{m}^{n} \quad \Xi_{n}^{m} \circ\left(1_{A^{\otimes(k)}} \otimes \sigma_{A, A} \otimes 1_{A^{\otimes(n-k-2)}}\right)=\Xi_{n}^{m} \quad \Xi_{1}^{1}=1_{A},
$$

where $\sigma_{A, A}: A \otimes A \rightarrow A \otimes A$ is the swap map, and, last but not least, that spiders which 'share' legs fuse together, i.e. spiders compose as follows:

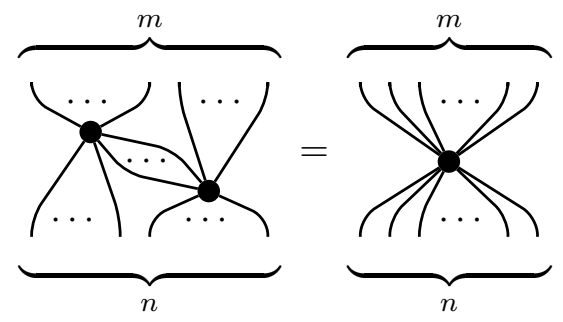

Conversely, the axioms of an internal commutative special dagger Frobenius algebra all follow from Eqs. (3.1) and (3.2). 
By compatibility of the classical structure with a given dagger compact structure we mean that for a spider on $A$ we have $\eta_{A}=\Xi_{2}^{0}$, and consequently that $\epsilon_{A}=\Xi_{0}^{2}$. In graphical terms, that is:

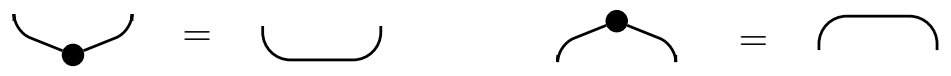

Indeed, for spiders $\Xi_{2}^{0}$ and $\Xi_{0}^{2}$ we always have:

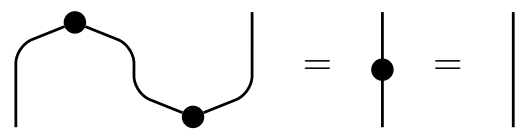

and

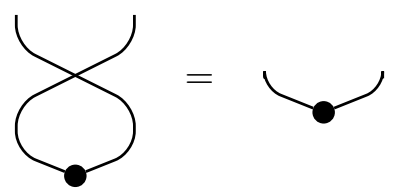

that is, they form a dagger compact structure.

In the graphical language we will depict elements (i.e. 'boxes without inputs') by triangles. By a pure classical element for a particular classical structure we mean an element which satisfies:

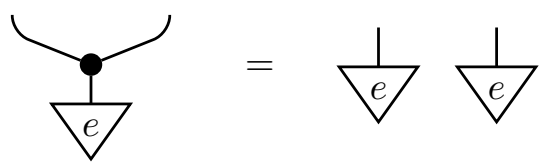

i.e. it is 'copied'. Below, by $e$ we will only denote such pure classical elements.

In the dagger compact category FHilb which has finite dimensional Hilbert spaces as objects, linear maps as morphisms, the tensor product as the monoidal structure, and adjoints as the dagger, classical structures are in bijective correspondence orthonormal bases via this concept of pure classical elements Coecke, Pavlovic and Vicary 12. Concretely, the pure classical elements are exactly the basis vectors, and conversely, given an orthonormal basis $\{|i\rangle\}_{i}$, the corresponding spiders are the linear maps with as only non-zero action on the basis vectors:

$$
\Xi_{n}^{m}:: \underbrace{|i \ldots i\rangle}_{n} \mapsto \underbrace{|i \ldots i\rangle}_{m}
$$

i.e. arrays of identical basis vectors are mapped on arrays of identical basis vectors, and all other basis vectors are mapped on the zero vector. Important particular examples are

$$
\Xi_{2}^{1}::|i j\rangle \mapsto \delta_{i j}|i\rangle \quad \text { and } \quad \Xi_{0}^{1}:: 1 \mapsto \sum_{i}|i\rangle
$$

which define the multiplication and its unit of the corresponding Frobenius algebra; their adjoints define the corresponding comultiplication and its counit.

Remark 3.4. In any dagger symmetric monoidal category, the multiplication and its unit suffice to specify a classical structure; one can then construct any other spider by composing $\Xi_{2}^{1}, \Xi_{0}^{1},\left(\Xi_{2}^{1}\right)^{\dagger}$ and $\left(\Xi_{0}^{1}\right)^{\dagger}$ to obtain a morphism with the required number of inputs $n$ and outputs $m$, that is, the spider $\Xi_{n}^{m}$. 
Remark 3.5. Physically relevant, rather than FHilb, is the category $W P(\mathbf{F H i l b})$ which is obtained by subjecting FHilb to the congruence which identifies those linear maps of the same type that are equal up to a complex phase, i.e.

$$
f \sim g \Leftrightarrow \exists \theta \in\left[0,2 \pi\left[: f=e^{i \theta} \cdot g .\right.\right.
$$

The reason is that vectors which are equal up to a complex phase represent the same state in quantum theory. The precise connection between classical structures in FHilb and those in $W P($ FHilb) is studied in detail in Coecke and Duncan 11]; roughly put -since this suffices for all practical purposes- classical structures are inherited.

\section{Complementary Classical structures}

Definition 4.1. A complementary endomorphism $H: A \rightarrow A$ for a classical structure is a (i) self-conjugate (ii) self-adjoint (iii) unitary endomorphism, graphically,

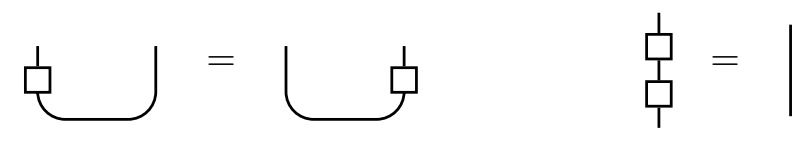

where self-adjointness is encoded in the symmetry of the small box depicting $H$, and, which 'transforms a given classical structure into a complementary one', which -following Coecke and Duncan 11] - graphically depicts as:

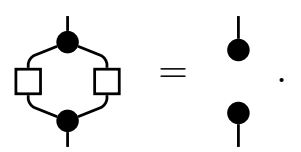

An example of such a complementary morphism is the familiar Hadamard matrix:

$$
\frac{1}{\sqrt{2}}\left(\begin{array}{rr}
1 & 1 \\
1 & -1
\end{array}\right)
$$

expressed in the basis defined by the classical structure. Rather than axiomatizing a pair of complementary classical structures as in [Coecke and Duncan 11, here we axiomatize a morphism which transforms a classical structure in the other one. The defining equation in Coecke and Duncan 11, which involves two classical structures, is obtained by postcomposing both sides of Eq. (4.1) with $H$, where

$$
\tilde{\Xi}_{2}^{1}:=H \circ \Xi_{2}^{1} \circ(H \otimes H) \quad \text { and } \quad \tilde{\Xi}_{0}^{1}:=H \circ \Xi_{0}^{1}
$$

then respectively are the multiplication and the unit of the second classical structure. Representing $\left(\Xi_{2}^{1}, \Xi_{0}^{1}\right)$ in green and $\left(\tilde{\Xi}_{2}^{1}, \tilde{\Xi}_{0}^{1}\right)$ in red, we have:

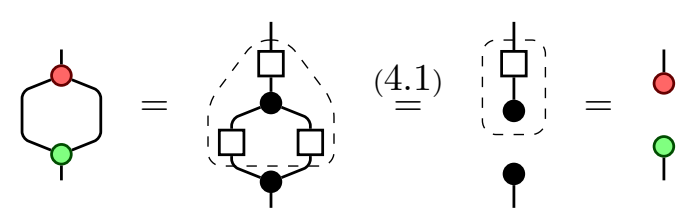

so we recover the characterization of complementarity as in Coecke and Duncan 11 $\S 8$. 
Remark 4.2. Since $H$ is self-transposed we can set

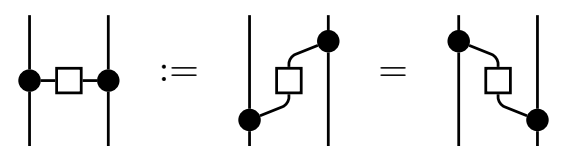

By Eq. (4.1) it then for example follows that:

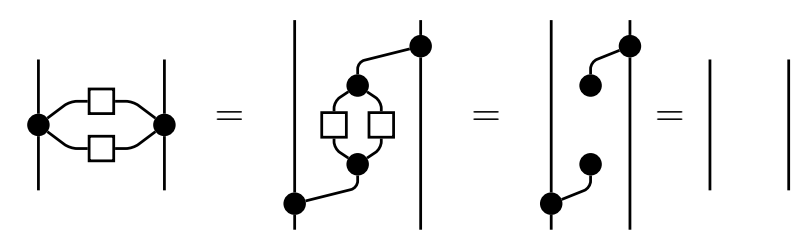

We will use this 'topological trick' throughout this paper.

\section{ENVIRONMENT}

We will consider two dagger compact categories, denoted $\mathbf{C}^{\text {pure }}$ and $\mathbf{C}$ respectively, and we assume that $\mathbf{C}^{\text {pure }}$ is a subcategory of $\mathbf{C}$ which inherits symmetric monoidal structure as well as dagger compact structure, and that $|\mathbf{C}|=\left|\mathbf{C}^{\text {pure }}\right|$.

Definition 5.1. An environment structure for $\left(\mathbf{C}^{\text {pure }}, \mathbf{C}\right)$ consists of a designated coelement $\top_{A}: A \rightarrow$ I for each object $A \in|\mathbf{C}|$, which we depicted as:

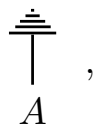

and that for all $A, B \in|\mathbf{C}|$ and all $f, g \in \mathbf{C}^{\text {pure }}(A, B)$ we have

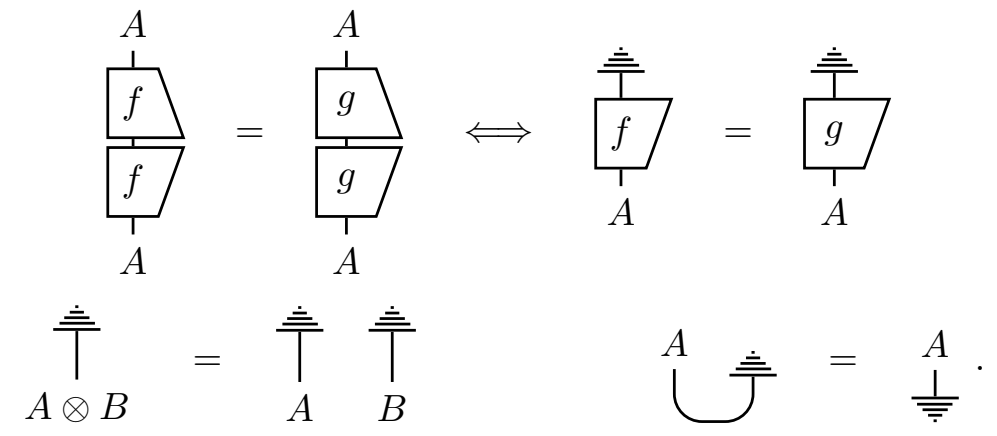

We will sometimes abbreviate environment structure to environment.

Remark 5.2. Eqs. (5.15.2) had already been introduced in [Coecke 08, as part of an axiomatization of mixed states and completely positive maps, but it was never considered in relation to classicality, measurements, and complementarity thereof. 
Example 5.3. Let Dens be the category with $\mathbb{C}^{n \times n}$ for $n \in \mathbb{N}$ as objects, and with completely positive maps $F: \mathbb{C}^{n \times n} \rightarrow \mathbb{C}^{m \times m}$ as morphisms. A morphism $f$ is pure, that is, $f \in$ hom $_{\text {Dens }}$ pure $\left(\mathbb{C}^{n \times n}, \mathbb{C}^{m \times m}\right)$, if there exists a linear map $L: \mathbb{C}^{n} \rightarrow \mathbb{C}^{m}$ such that $f:: \rho \mapsto L \rho L^{\dagger}$. Then, the usual trace

$$
\top_{\mathbb{C}^{n \times n}}: \mathbb{C}^{n \times n} \rightarrow \mathbb{C}:: \rho \mapsto \operatorname{tr}(\rho)
$$

provides an environment structure for $\left(\right.$ Dens $^{\text {pure }}$, Dens). Indeed, for any $f:: \rho \mapsto L \rho L^{\dagger}$ and $g:: \rho \mapsto M \rho M^{\dagger}$, equation 5.1 is satisfied:

$$
\begin{aligned}
f^{\dagger} \circ f=g^{\dagger} \circ g & \Longleftrightarrow \forall \rho: L^{\dagger}\left(L \rho L^{\dagger}\right) L=M^{\dagger}\left(M \rho M^{\dagger}\right) M \\
& \Longleftrightarrow L^{\dagger} L=M^{\dagger} M \\
& \Longleftrightarrow \forall \rho: \operatorname{tr}\left(L^{\dagger} L \rho\right)=\operatorname{tr}\left(M^{\dagger} M \rho\right) \\
& \Longleftrightarrow \forall \rho: \operatorname{tr}\left(L \rho L^{\dagger}\right)=\operatorname{tr}\left(M \rho M^{\dagger}\right) \\
& \Longleftrightarrow \top \circ f=\top \circ g .
\end{aligned}
$$

This example justifies the name 'environment': tracing a system out in quantum theory is interpreted as this system being part of the environment.

Example 5.4. Let $\mathbf{C}$ be any dagger compact category, let $C P M(\mathbf{C})$ the category obtained by applying Selinger's CPM-construction [Selinger 07] -we explicitly present this construction in Section 8 - and let $W P(\mathbf{C})$ be the category obtained by applying the 'doubling construction' of [Coecke 07] which cancels out 'abstract global phases' -cf. Remark 3.5. Then, the caps of the dagger compact structure provide an environment for $(W P(\mathbf{C}), C P M(\mathbf{C}))$ Coecke 08. When taking $\mathbf{C}$ to be FHilb then we recover the previous example, with:

$$
\text { Dens }=C P M(\text { FHilb }) \quad \text { and } \quad \text { Dens }^{\text {pure }}=W P(\text { FHilb }) .
$$

Below we assume as given a pair $\left(\mathbf{C}^{\text {pure }}, \mathbf{C}\right)$ with an environment. We set

$$
\perp_{A}:=\top_{A}^{\dagger}: \mathrm{I} \rightarrow A \text {. }
$$

An element $\psi: \mathrm{I} \rightarrow A$ is normalized if

$$
\top_{A} \circ \psi=1_{\mathrm{I}} .
$$

Below all elements depicted as triangles are normalized, diagrammatically:

$$
\frac{\dot{\overline{1}}}{\Downarrow}=
$$

where $1_{I}$ is graphically represented by an empty picture.

Proposition 5.5. A morphism $f \in \mathbf{C}^{\text {pure }}$ is an isometry iff $\top_{B} \circ f=\top_{A}$, and hence, it is unitary iff we moreover have that $f \circ \perp_{A}=\perp_{B}$.

\section{Classical channels, measurements and Classical control}

Definition 6.1. Let $\Xi$ be a classical structure. The morphism:

$$
C_{\Xi}=
$$

is called the classical channel of type $\Xi$. 
In the light of the discussion in Section 2, this picture can be interpreted as 'copying into the environment', that is, 'broadcasting', or in the decoherence view, 'being coupled to the environment'.

Example 6.2. In Dens, for the classical structure of Eqs. (3.5), we have

$$
C_{\Xi}(\rho)=\operatorname{tr}\left(\Xi_{1}^{2} \circ \rho \circ \Xi_{2}^{1}\right)=\mathcal{D}_{\{|i\rangle\}_{i}}
$$

where $\mathcal{D}_{\{|i\rangle\}_{i}}$ was defined in Eq. (2.3). That is, a classical channel preserves the diagonal data relative to the basis that is specified by its basis structure. It will however destroy the non-diagonal data. In the light of the discussion of Section 2, it is a 'classicizing' operation, on the data it is applied to.

Remark 6.3. While physically all classical channels are of course the same, our classical channels in addition carry specification of how the classical data it transmits has been obtained, in terms of a dependency on the classical structure $\Xi$ which specifies a particular quantum measurement. In the light of the fact that by default we take all systems to be quantum, this specification of the classical structure relative to which classical data is classical is indeed unavoidable. It is for this reason that we choose to axiomatize the complementary morphism -cf. the discussion in Section 4- which enables us to restrict ourselves to a single classical structure.

The following proposition shows that a classical channel leaves its pure classical elements invariant, and that it is idempotent. In fact, we could define more general classical elements $p: \mathrm{I} \rightarrow A$ as those that satisfy

$$
C_{\Xi} \circ p=p \text {. }
$$

Physically, this means that a classical channel 'transmits' its classical elements. Equivalently, classical elements are invariant under decoherence.

\section{Proposition 6.4.}
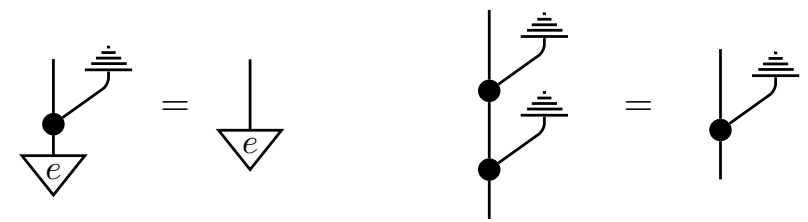

Proof. The first equality follows from Eq. (3.3) and Eq. (5.5), and

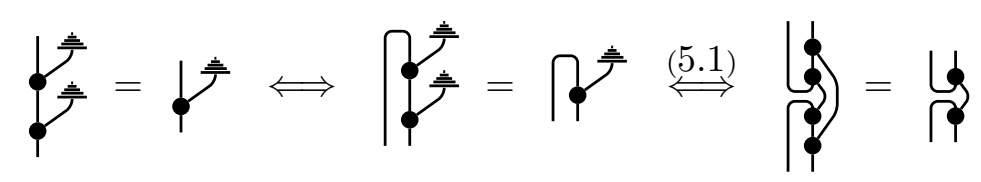

where the last equality holds due to the spider normal form theorem.

We can now construct a measurement as follows:

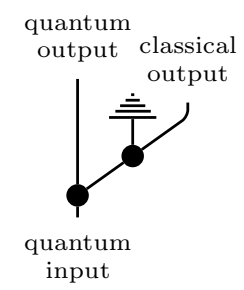


i.e. it copies the quantum data into a classical channel. A destructive measurement is obtained by 'feeding the quantum output itself into the environment'. Proposition 6.4 then yields:

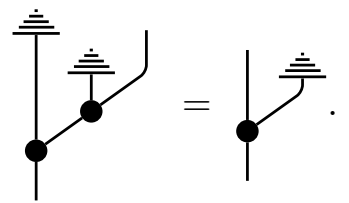

and the resulting shape of the destructive measurement is then:

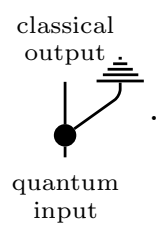

Note here in particular that destructive measurements and classical channels are 'semantically equivalent'. Similarly, by the spider normal form we have:

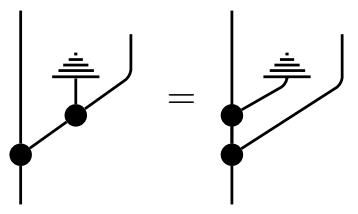

so the quantum output of a measurement is 'semantically equivalent' to its classical output, which captures change of the quantum state to an eigenstate. More generally, as a consequence of the structural power of the spider normal form theorem, classicality 'semantically spreads through a diagram'.

Example 6.5. We now illustrate the above exposed diagrammatic analysis on the concrete example of measurement of a qubit. For $|\psi\rangle=\psi_{0}|0\rangle+\psi_{1}|1\rangle$ to which we apply the morphism of of Eq. (6.2) which we assume to be in the computational basis, the first 'copying' operation yields $\psi_{0}|00\rangle+\psi_{1}|11\rangle$, the second one yields $\psi_{0}|000\rangle+\psi_{1}|111\rangle$ and the effect of the environment yields, now necessarily in density matrix terms, $\left|\psi_{0}\right|^{2}|00\rangle\left\langle\left. 00|+| \psi_{1}\right|^{2} \mid 11\right\rangle\langle 11|$.

Note that by idempotence of $C_{\Xi}$ it also follows that $\perp_{A}=\top_{A}^{\dagger}$ is a classical element, and in particular, that this does not depend on the choice of $\Xi$. We will call $\perp_{A}$ (unnormalized) maximal mixedness.

Example 6.6. In Dens we indeed have that $\perp_{\mathcal{H}}$ is diagonal in any basis.

We call a morphism $f: A \rightarrow B$ disconnected if it factors along I, that is, if $f=\psi \circ \pi$ for some $\psi: \mathrm{I} \rightarrow B$ and $\pi: A \rightarrow \mathrm{I}$. In the graphical representation we indeed obtain a disconnected picture in this case:

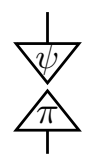

The topological disconnectedness physically stands for the fact that there is no information flowing from the input to the output. 
Remark 6.7. For non-trivial categories the morphisms $\top_{A}$ cannot be pure; if they would be pure then setting $f:=\top_{A}$ and $g:=1_{A}$ in Eq. 5.1, the righthandside becomes $1_{\mathrm{I}} \circ \top_{A}=$ $\top_{A} \circ 1_{A}$, which holds, and hence also the lefthandside holds: $\perp_{A} \circ \top_{A}=1_{A} \circ 1_{A}=1_{A}$. That is, the identity is 'disconnected'. This is obviously in conflict with the intuition that through a straight wire information flows without being modified, so one expects bad things to happen. Indeed, for any $f: A \rightarrow B$ we now have $f=1_{B} \circ f \circ 1_{A}=\perp_{B} \circ \top_{B} \circ f \circ \perp_{A} \circ \top_{A}=s \cdot \perp_{B} \circ \top_{A}$ with $s=\top_{B} \circ f \perp_{A}: \mathrm{I} \rightarrow \mathrm{I}$ a scalar. That is, any morphism is disconnected!

If we introduce $H$ between $C_{\Xi}$ and itself, we obtain 'complementary behaviors'. The first equality of the following proposition implies that a measurement turns a pure classical element of a complementary measurement in maximal mixedness, i.e. any outcome is equally probable for that measurement (cf. 'unbiasedness'). The second one implies that there is no dataflow from the input to the output when we compose complementary measurements.

Proposition 6.8.
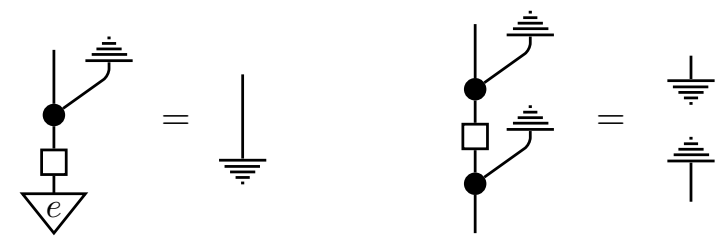

Proof. We have:

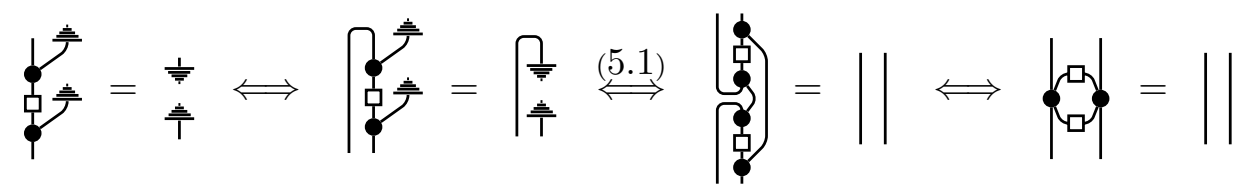

The first equation is derived from the second one and Proposition 6.4:

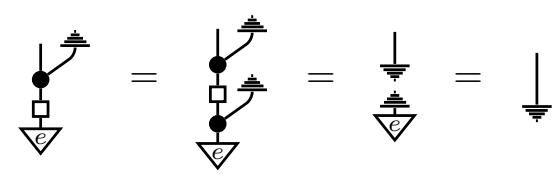

6.1. General classical control operations. In diagrammatic terms, a morphism $U$ : $A \rightarrow A$ is unitary if:

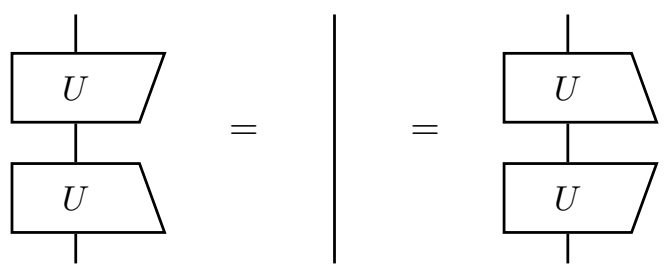

We now define what it means to have a family of unitaries of the same type, 'indexed' by a classical structure, that is, a controlled unitary. 
Definition 6.9. By a controlled unitary we mean an operation of the form:

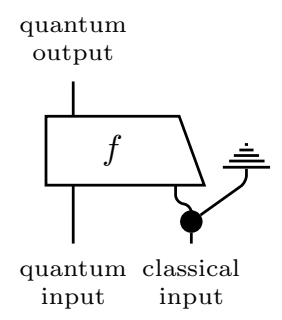

which 'for all classical input values is unitary', that is:

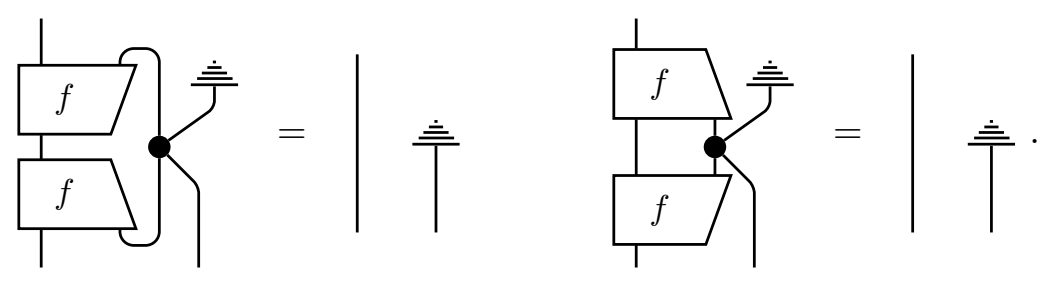

Lemma 6.10. The following morphisms are controlled unitaries:

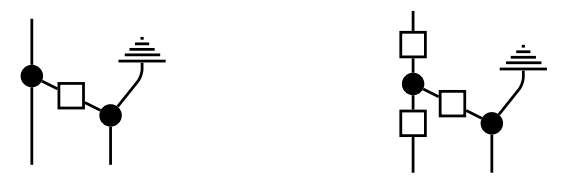

Proof. We have:

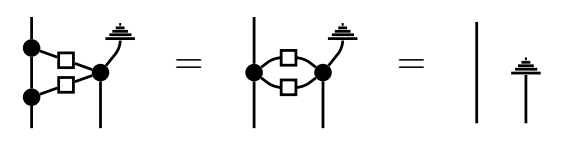

and the remainder of the proof proceeds almost identical.

6.2. General non-degenerate measurements. We have identified an example of a nondegenerate measurement, namely the one of the shape (6.2), and an example of a nondegenerate destructive measurement, namely the one of the shape (6.3). Relative to a given classical structure we can define more general non-destructive measurements.

The following Lemma shows how classical data can be composed in terms of classical structures, where we conceive classical structures as being specified by a multiplication and its unit, i.e. $\Xi:=\left(\Xi_{2}^{1}, \Xi_{0}^{1}\right)$-cf. Remark 3.4 .

Lemma 6.11. If $\left(\Xi_{2}^{1}, \Xi_{0}^{1}\right)$ and $\left(\tilde{\Xi}_{2}^{1}, \tilde{\Xi}_{0}^{1}\right)$ are classical structures on $A$ and $\tilde{A}$ respectively, then the morphisms

$$
\begin{gathered}
\left(\Xi_{2}^{1} \otimes \tilde{\Xi}_{2}^{1}\right) \circ\left(1_{A} \otimes \sigma_{A, \tilde{A}} \otimes 1_{\tilde{A}}\right):(A \otimes \tilde{A}) \otimes(A \otimes \tilde{A}) \rightarrow A \otimes \tilde{A} \\
\Xi_{0}^{1} \otimes \tilde{\Xi}_{0}^{1}: \mathrm{I} \rightarrow A \otimes \tilde{A}
\end{gathered}
$$


define a classical structure on $A \otimes \tilde{A}$, diagrammatically,

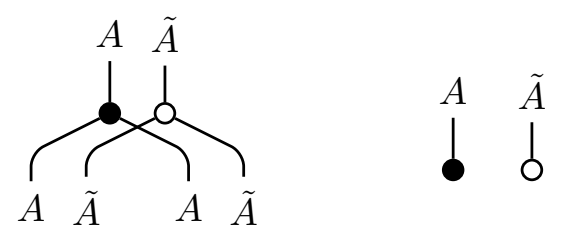

The canonically corresponding non-degenerate destructive measurement which extracts this compound classical data from a pair of quantum systems is:

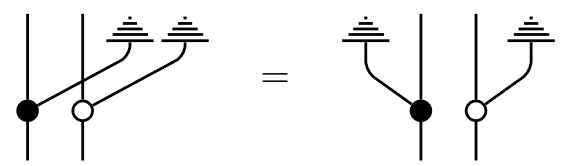

When transforming the quantum data by means of a unitary we obtain the general form of a non-degenerate distructive measurement on a pair of systems:

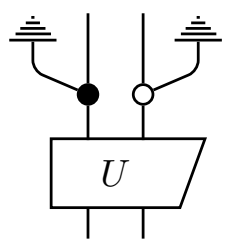

Definition 6.12. Given a classical structure on $A$, a non-degenerate measurement on $n$ systems of type $A$ is a morphism of the form:

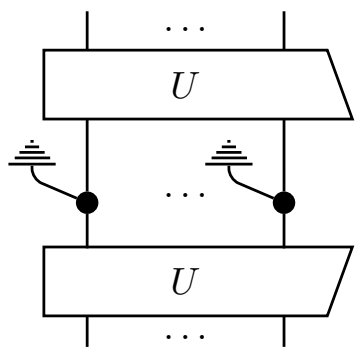

where $U: A \otimes \ldots \otimes A \rightarrow A \otimes \ldots \otimes A$ is an arbitrary unitary, and a corresponding nondegenerate destructive measurement is a morphism of the form:

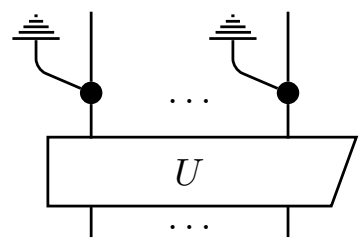

Remark 6.13. One can also define more general kinds of measurements, namely degenerate ones and non-projective ones, which involves defining projective measurements without reference to unitaries. This can be done in straightforward analogy to how this was done in [Coecke and Pavlovic 07] and Coecke, Paquette and Pavlovic 09. 
Lemma 6.14. The following morphism is unitary:<smiles>CC(C)C(Cl)OI</smiles>

Proof.

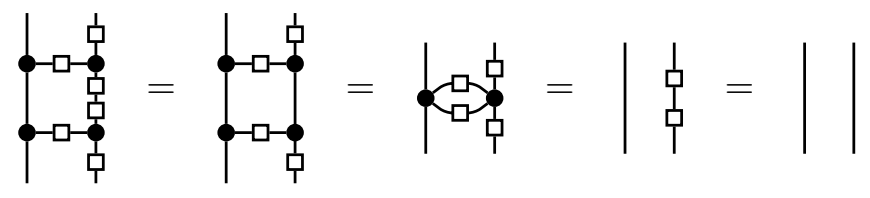

Corollary 6.15. The following morphism is a non-degenerate destructive measurement on a pair of systems of the same type A:

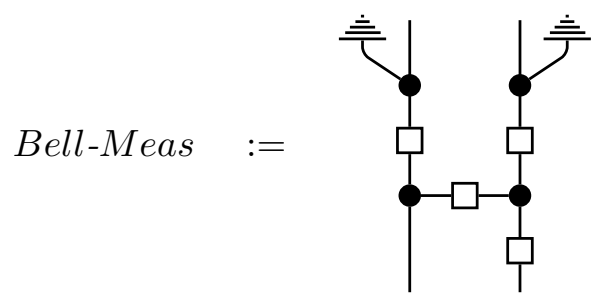

6.3. Interpretation of graphical elements in Dens. The following tables translate the graphical language to Hilbert space quantum theory for the specific case of qubits. It is this translation which connects that diagrammatic presentation of the protocols in the following section to how one finds them usually described in textbooks.

\begin{tabular}{|c|c|c|c|c|}
\hline Notation: & $d$ & 9 & $\downarrow$ & 9 \\
\hline Dens $^{\text {pure }}:$ & $1 \mapsto 2 \cdot|+\rangle\langle+|$ & $\rho \mapsto 2 \cdot\langle+|\rho|+\rangle$ & $1 \mapsto 2 \cdot|0\rangle\langle 0|$ & $\rho \mapsto 2 \cdot\langle 0|\rho| 0\rangle$ \\
\hline \multicolumn{5}{|l|}{ Notation: } \\
\hline Dens $^{\text {pure }}$ : & $1 \mapsto(|00\rangle+\mid 1$ & \rangle$)(\langle 00|+\langle 11|)$ & $\rho \mapsto(\langle 00|+\langle 11$ & $\rho(|00\rangle+|11\rangle)$ \\
\hline
\end{tabular}


(pure) gates:

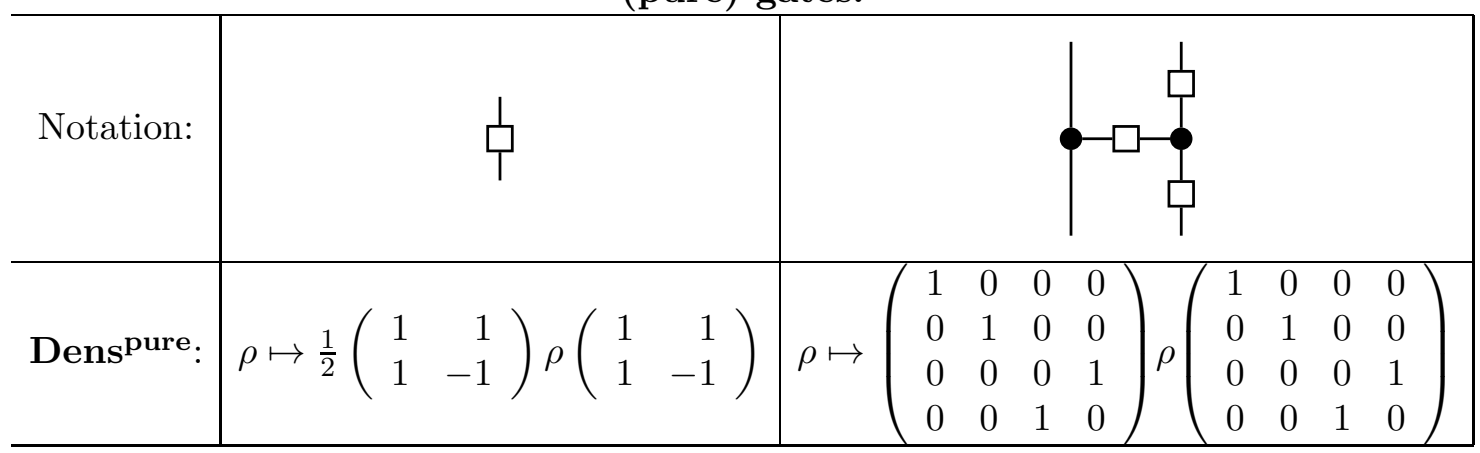

\section{CP maps:}

\begin{tabular}{|c|c|c|c|}
\hline Notation: & $\underline{\underline{\underline{ }}}$ & & \\
\hline Dens: & $\begin{array}{c}\text { trace } \\
\rho \mapsto \operatorname{tr}(\rho)\end{array}$ & $\begin{array}{c}\text { maximally } \\
\text { mixed state } \\
1 \mapsto \frac{1}{2}\left(\begin{array}{ll}1 & 0 \\
0 & 1\end{array}\right)\end{array}$ & $\begin{array}{c}\text { erase } \\
\text { non-diagonal elements } \\
\rho \mapsto\langle 0|\rho| 0\rangle+\langle 1|\rho| 1\rangle\end{array}$ \\
\hline
\end{tabular}

(destructive) measurements:

\begin{tabular}{c|c|c|c}
\hline Notation: & & &
\end{tabular}

classically controlled operations:

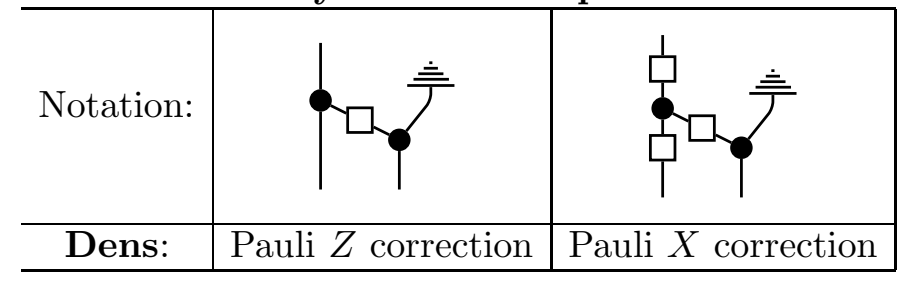

\section{ExAmple protocols}

In the statement of each proposition, we will specify protocols with explicit physical types, quantum channels being represented by full lines and classical channels being represented by dotted lines. We use the symbol ' $: \simeq$ ' for the passage of this specification to the interpretation within the diagrammatic calculus. 
First we show that the teleportation protocol, by means of a Bell state and two classical channels, realizes a (perfect) quantum channel.

Proposition 7.1 (correctness of teleportation).
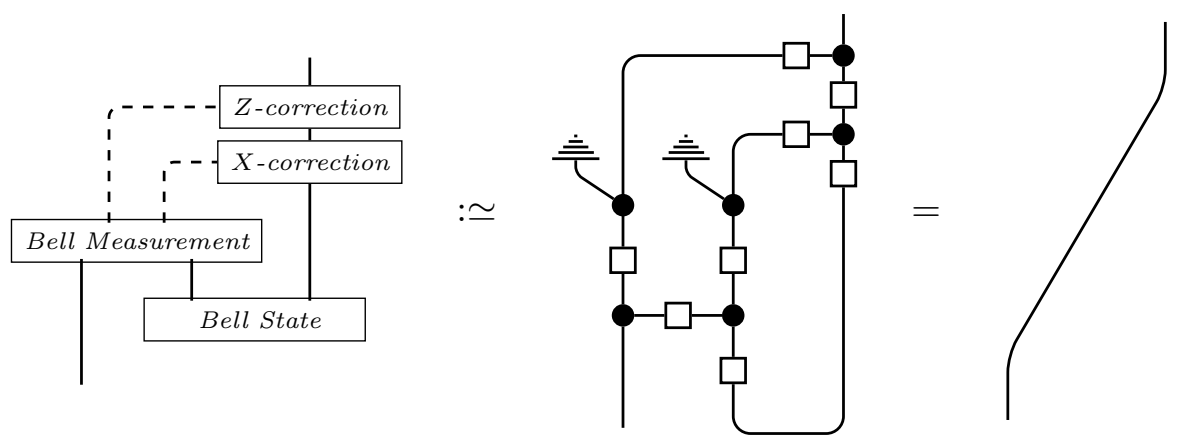

Proof.

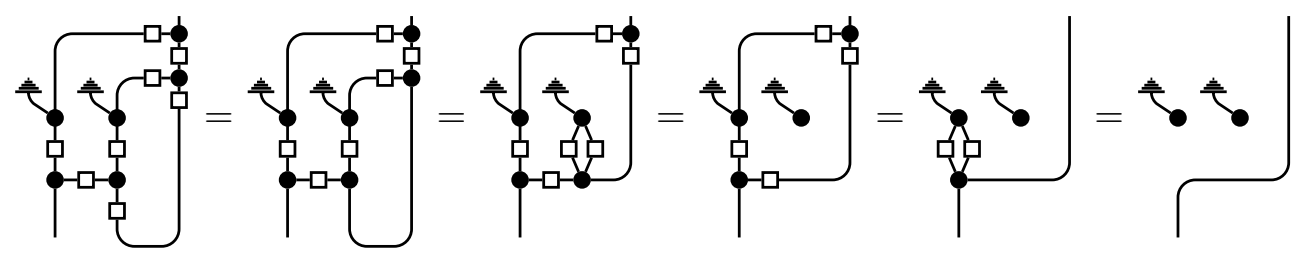

We show that the state transfer Perdrix 05] protocol, by means of 2-qubit unitary tranformation and a local measurement, realizes a (perfect) quantum channel.

Proposition 7.2 (correctness of state transfer).
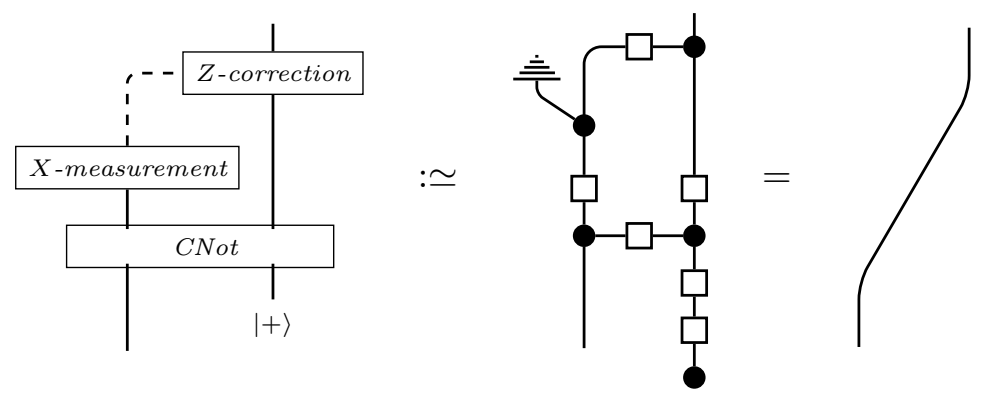

Proof.

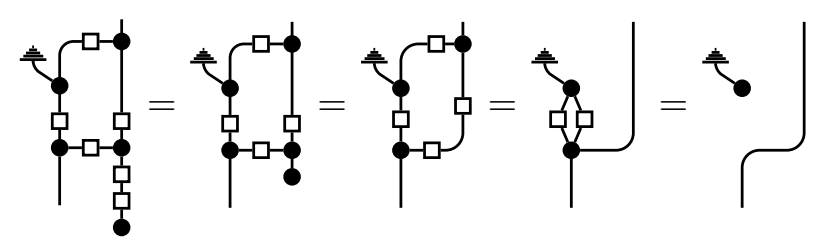


Now we show that the dense coding protocol, by means of a Bell state and a quantum channel, realizes two classical channels.

Proposition 7.3 (correctness of dense coding).
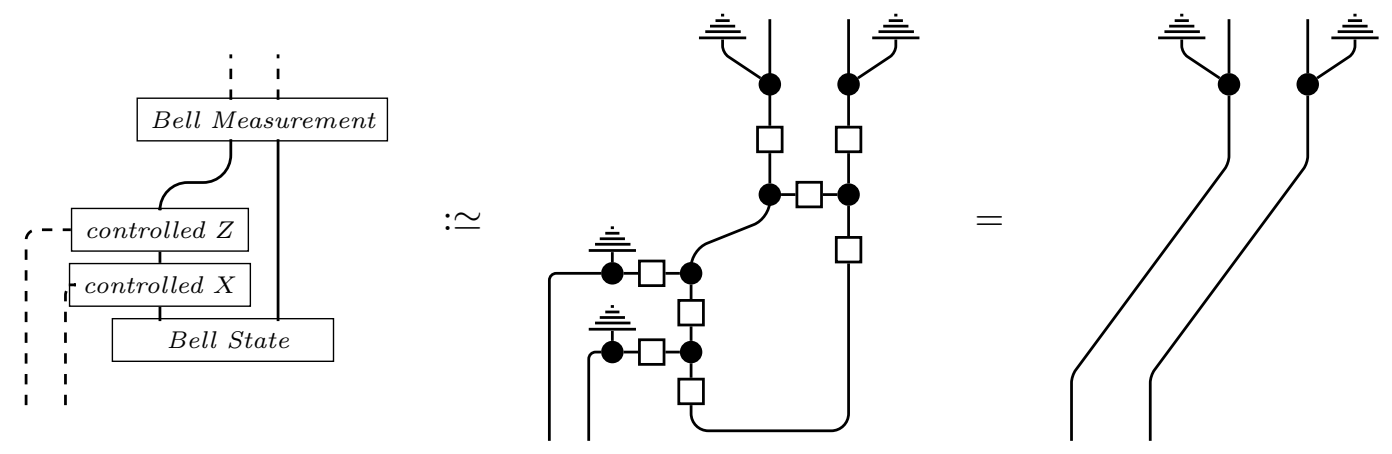

Proof.

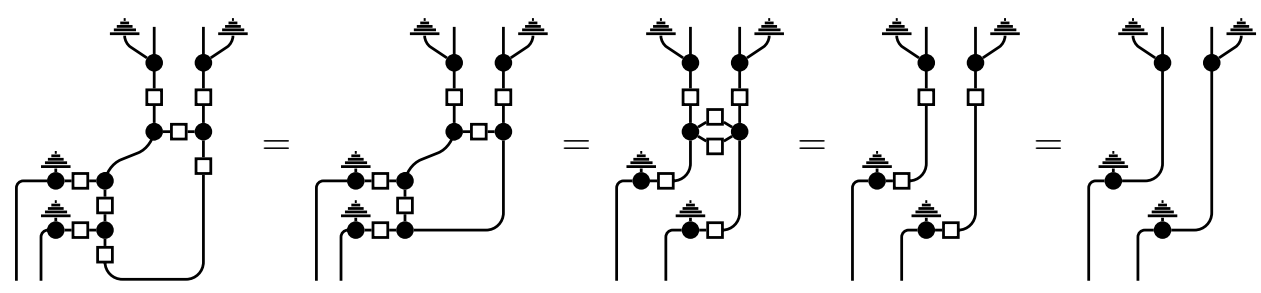

A diagrammatic presentation of a quantum key exchange protocols can be found in Coecke, Wang (x3) and Zhang 11. Here we provide a simplified presentation by relying on the notion of environment. We restrict ourselves to four representative cases.

Proposition 7.4 (correctness of BB84 and Ekert 91 key exchange).

- Alice and Bob choose the same measurement in Ekert 91:

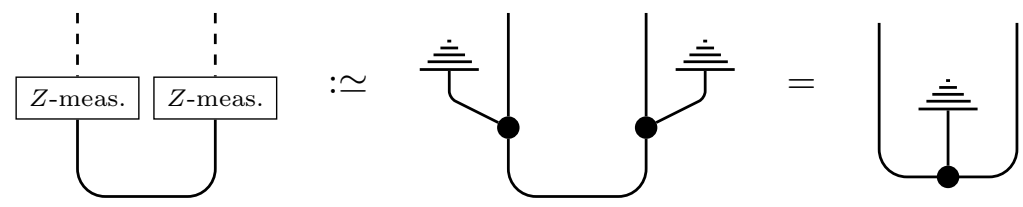

i.e. Alice and Bob share the same classical data.

- Alice and Bob choose a different measurement in Ekert 91:

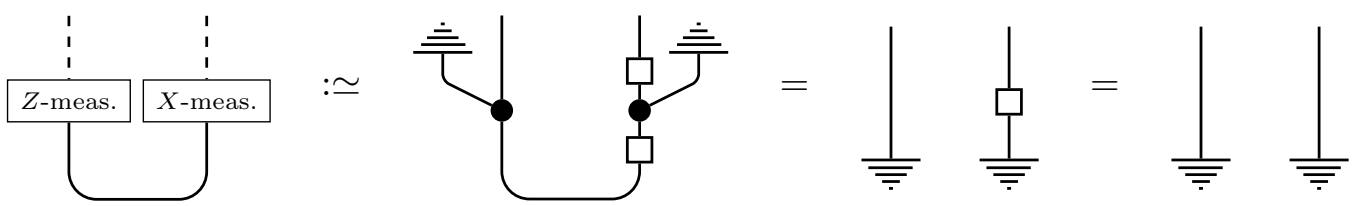

i.e. Alice's and Bob's data is not correlated. 
- Eve chooses the same measurement as Alice and Bob in BB84:
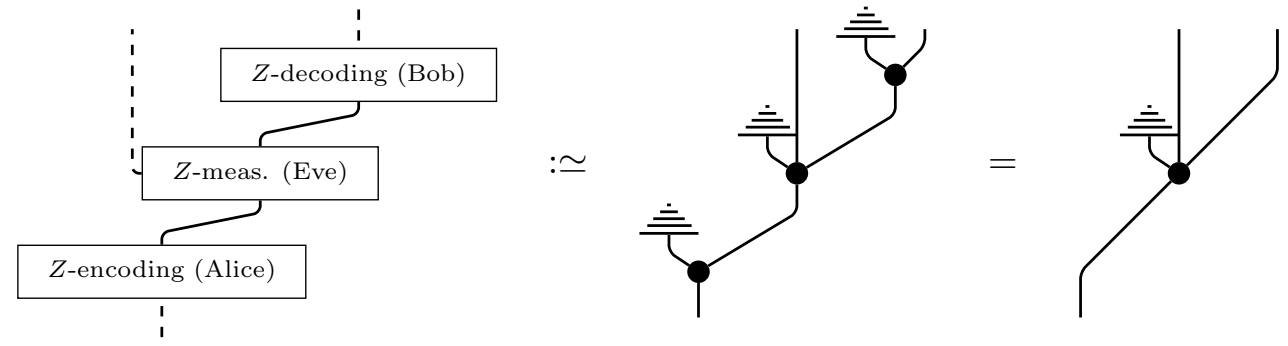

i.e. Alice, Bob and Eve share the same classical data.

- Eve chooses a different measurement than Alice and Bob in BB84:
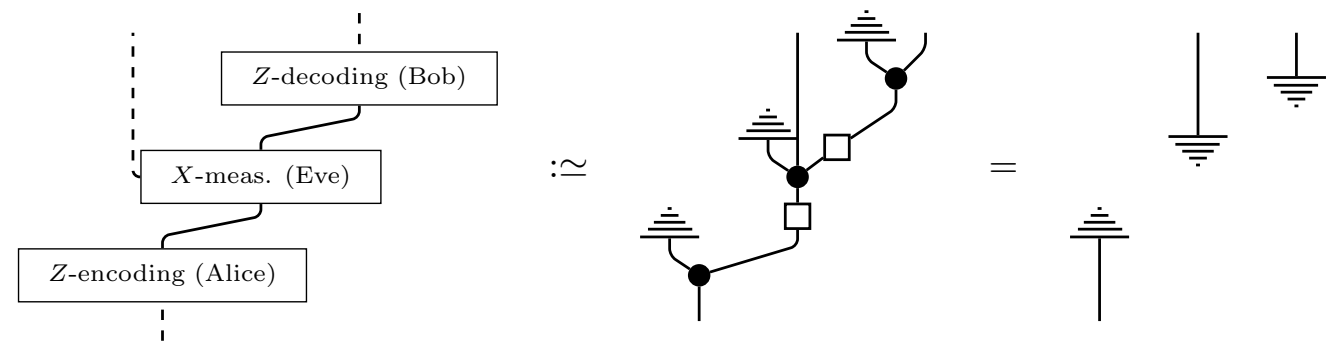

i.e. Alice's, Bob's and Eve's data is not correlated.

\section{Connection to Selinger's CPM-Construction}

Here we briefly describe the connection between Definition 5.1 and the CPM-comstruction [Selinger 07], which was established in [Coecke 08]. Given any dagger compact category $\mathbf{C}$, we define a new category $C P M(\mathbf{C})$ which has the same objects as $\mathbf{C}$, and a morphisms of type $A \rightarrow B$ in $C P M(\mathbf{C})$ is a morphism of type $A \otimes A \rightarrow B \otimes B$ in $\mathbf{C}$ of the shape:

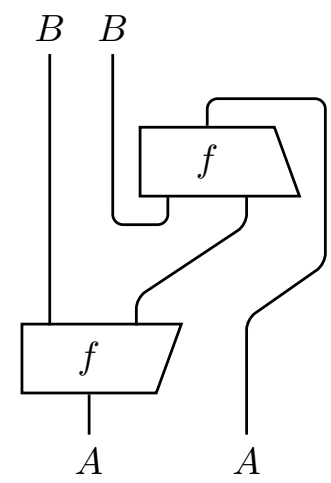

where $f: A \rightarrow B \otimes C$ is any morphism in $\mathbf{C}$. Then, $C P M(\mathbf{C})$ is again a dagger compact category, and as already mentioned in Example 5.4, if we set $\mathbf{C}:=$ FHilb then the morphisms of the form (8.1) are exactly completely positive maps. 
Definition 8.1. An environment with purification for $\left(\mathbf{C}^{\text {pure }}, \mathbf{C}\right)$ is an environment as in Definition 5.1 for which we in addition have that, denoting morphisms in $\mathbf{C}^{\text {pure }}$ and more general morphisms in $\mathbf{C}$ respectively as

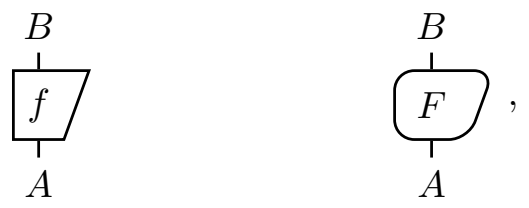

that for all $A, B \in|\mathbf{C}|, F \in \mathbf{C}(A, B)$ there exists $f \in \mathbf{C}^{\text {pure }}(A, B \otimes C)$ such that

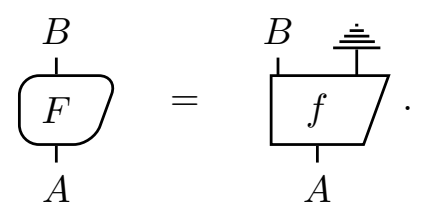

Theorem 8.2. Coecke 08] $C P M\left(\mathbf{C}^{\text {pure }}\right)=\mathbf{C}$.

As already mentioned in Example 5.4, the converse statement, that for any dagger compact category $\mathbf{C}$ the category $C P M(\mathbf{C})$ provides an environment with purification also holds, up to a minor and physically justified assumption related to the fact that vectors which are equal up to a complex phase represent the same state in quantum theory. Concretely, this axiom states the for all pure elements $\psi, \psi^{\prime}: \mathrm{I} \rightarrow A$ we have:

$$
\psi \circ \psi^{\dagger}=\psi^{\prime} \circ \psi^{\prime \dagger} \Rightarrow \psi=\psi^{\prime} \text {. }
$$

This equation follows from Eq. (5.1) when setting $f:=\psi^{\dagger}$ and $g:=\psi^{\prime \dagger}$.

Remark 8.3. The power of purification as an axiom for quantum theory has recently been exploited in Chiribella, D'Ariano and Perinotti 09, Chiribella, D'Ariano and Perinotti 10, although there, the authors also require certain uniqueness properties.

\section{Conclusion}

An axiomatization of the concept of environment resulted in a very simple comprehensive graphical calculus, which in particular enables one to reason about classical-quantum interaction in quantum informatic protocols.

Several operationally distinct concepts turn out to have the same semantics within the graphical language (e.g. classical channel, measurement, preparation as in BB84). Consequently, all that one structurally truly needs are Propositions 6.4 and 6.8 on composition of classical channels and pure classical elements.

The examples given here are simple but representative. This work and the earlier contributions on which we relied together successfully addresses a challenge for the categorical quantum mechanics research program which was set at the very beginning: to have a very simple graphical description of all basic quantum informatic protocols, in particular including classical-quantum interaction.

The new graphical element 'environment' and the interaction rules for classical channels can now be integrated in the quantomatic software, so that it can now be used to (semi)automate reasoning about full-blown quantum informatic protocols, including classicalquantum interaction. 
Here we only considered two complementary observables, and no phase data. We meanwhile also have several graphical calculi that are universal for quantum computing Coecke and Duncan 11, Coecke and Kissinger 10. The next step of this research strand would be to extend the graphical calculus presented here to these calculi, which include, for example, phases and $W$-states.

This work could also be advanced in the direction of quantum information theory. In particular, one may want to study whether it would be possible to obtain a diagrammatic account on quantum informatic quantities. Some examples of diagrammatic quantum informatic quantities are in Coecke 08.

\section{ACKNOWLEDGEMENTS}

Work supported by EP/D072786/1, ONR N00014-09-1-0248 and EU FP6 STREP QICS. John Baez, Aleks Kissinger, Prakash Panangaden, Johan Paulsson, Jamie Vicary, the CSL'10 referees and the LMCS referees provided useful feedback on an earlier versions.

\section{REFERENCES}

[Abramsky 09] S. Abramsky (2009) No-cloning in categorical quantum mechanics. In: Semantic Techniques for Quantum Computation, I. Mackie and S. Gay (eds), pages 1-28, Cambridge University Press. arXiv:0910.2401

[Abramsky and Coecke 04] S. Abramsky and B. Coecke (2004) A categorical semantics of quantum protocols. In: Proceedings of 19th IEEE conference on Logic in Computer Science (LiCS), pages 415-425. IEEE Press. arXiv:quant-ph/0402130. Revised version (2009): Categorical quantum mechanics. In: Handbook of Quantum Logic and Quantum Structures, K. Engesser, D. M. Gabbay and D. Lehmann (eds), pages 261-323. Elsevier. arXiv:0808.1023

[Abramsky and Coecke 05] S. Abramsky and B. Coecke (2005) Abstract physical traces. Theory and Applications of Categories 14, 111-124. arXiv:0910.3144

[Barnum et al. 96] H. Barnum, C. M. Caves, C. A. Fuchs, R. Jozsa, and B. Schumacher (1996) Noncommuting mixed states cannot be broadcast. Physical Review Letters 76, 2818-2821. arXiv:quant-ph/9511010 [Bennett and Brassard 84] C. H. Bennett and G. Brassard (1984) Quantum cryptography: Public key distribution and coin tossing. In: Proceedings of IEEE International Conference on Computers, Systems and Signal Processing, pages 175-179.

[Bennett et al. 93] C. H. Bennett, G. Brassard, C. Crépeau, R. Jozsa, A. Peres and W. K. Wooters (1993) Teleporting an unknown quantum state via dual classical and Einstein-Podolsky-Rosen channels. Physical Review Letters 70, 1895-1899.

[Bennett and Wiesner 92] C. H. Bennett and S. Wiesner (1992) Communication via one- and two-particle operators on Einstein-Podolsky-Rosen states. Physical Review Letters 69, 2881-2884.

[Carboni and Walters 87] A. Carboni and R. F. C. Walters (1987) Cartesian bicategories I. Journal of Pure and Applied Algebra 49, 11-32.

[Chiribella, D'Ariano and Perinotti 09] G. Chiribella, G. M. D'Ariano and P. Perinotti (2009) Probabilistic theories with purification. Physical Review A 81, 062348. arXiv:0908.1583

[Chiribella, D'Ariano and Perinotti 10] G. Chiribella, G. M. D'Ariano, and P. Perinotti (2010) Informational derivation of quantum theory. arXiv:1011.6451

[Coecke 07] B. Coecke (2007) De-linearizing linearity: projective quantum axiomatics from strong compact closure. Electronic Notes in Theoretical Computer Science 170, 47-72. arXiv:quant-ph/0506134

[Coecke 08] B. Coecke (2008) Axiomatic description of mixed states from Selinger's CPM-construction. Electronic Notes in Theoretical Computer Science 210, 3-13.

[Coecke and Duncan 08] B. Coecke and R. Duncan (2008) Interacting quantum observables. In: Proceedings of the 35th International Colloquium on Automata, Languages and Programming (ICALP), pp. 298-310, Lecture Notes in Computer Science 5126, Springer-Verlag. 
[Coecke and Duncan 11] B. Coecke and R. Duncan (2011) Interacting quantum observables: categorical algebra and diagrammatics. New Journal of Physics 13, 043016. arXiv:0906.4725.

[Coecke, Edwards and Spekkens 11] B. Coecke, B. Edwards and R. W. Spekkens (2011) Phase groups and the origin of non-locality for qubits. Electronic Notes in Theoretical Computer Science 270 (2), 15-36. arXiv:1003.5005

[Coecke and Kissinger 10] B. Coecke and A. Kissinger (2010) The compositional structure of multipartite quantum entanglement. In: Proceedings of the 37th International Colloquium on Automata, Languages and Programming (ICALP), pp. 297-308, Lecture Notes in Computer Science 6199, Springer-Verlag. arXiv:1002.2540

[Coecke and Paquette 11] B. Coecke and E. O. Paquette (2011) Categories for the practicing physicist. In: New Structures for Physics, B. Coecke (ed), pages 167-271. Lecture Notes in Physics, Springer-Verlag. arXiv:0905.3010

[Coecke, Paquette and Perdrix 08] B. Coecke, E. O. Paquette and S. Perdrix (2008) Bases in diagrammatic quantum protocols. Electronic Notes in Theoretical Computer Science 218, 131-152. arXiv:0808.1037

[Coecke, Paquette and Pavlovic 09] B. Coecke, E. O. Paquette and D. Pavlovic (2009) Classical and quantum structuralism. In: Semantic Techniques for Quantum Computation, I. Mackie and S. Gay (eds), pages 29-69, Cambridge University Press. arXiv:0904.1997

[Coecke and Pavlovic 07] B. Coecke and D. Pavlovic (2007) Quantum measurements without sums. In: Mathematics of Quantum Computing and Technology, G. Chen, L. Kauffman and S. Lamonaco (eds), pages 567-604. Taylor and Francis. arXiv:quant-ph/0608035.

[Coecke, Pavlovic and Vicary 12] B. Coecke, D. Pavlovic, and J. Vicary (2012) A new description of orthogonal bases. Mathematical Structures in Computer Science, to appear. arXiv:0810.0812

[Coecke, Wang (x3) and Zhang 11] B. Coecke, B.-S. Wang, Q.-L. Wang, Y.-J. Wang and Q.-Y. Zhang (2011) Graphical calculus for quantum key distribution. Electronic Notes in Theoretical Computer Science 270 (2), 231-249.

[Dieks 82] D. G. B. J. Dieks (1982) Communication by EPR devices. Physics Letters A 92, 271-272.

[quantomatic] L. Dixon, R. Duncan, B. Frot, A. Kissinger, A. Merry, M. Soliviev (2011) quantomatic software tool, http://sites.google.com/site/quantomatic/

[Dixon and Kissinger 11] L. Dixon and A. Kissinger (2011) Open graphs and monoidal theories. Mathematical Structures in Computer Science, to appear. arXiv:1011.4114

[Duncan and Perdrix 09] R. Duncan and S. Perdrix (2009) Graph states and the necessity of Euler decomposition. In: Proceedings of Computability in Europe: Mathematical Theory and Computational Practice (CiE'09), pages 167-177. Lecture Notes in Computer Science 5635, Springer-Verlag. arXiv:0902.0500

[Duncan and Perdrix 10] R. Duncan and S. Perdrix (2010) Rewriting measurement-based quantum computations with generalised flow. In: Proceedings of the 37th International Colloquium on Automata, Languages and Programming (ICALP), Lecture Notes in Computer Science 6199, Springer-Verlag.

[Ekert 91] A. Ekert (1991) Quantum cryptography based on Bell's theorem. Physical Review Letters 67, 661-663.

[Joyal and Street 91] A. Joyal and R. Street (1991) The Geometry of tensor calculus I. Advances in Mathematics 88, 55-112.

[Kelly and Laplaza 80] G. M. Kelly and M. L. Laplaza (1980) Coherence for compact closed categories. Journal of Pure and Applied Algebra 19, 193-213.

[Lack 04] S. Lack (2004) Composing PROPs. Theory and Applications of Categories 13, 147-163.

[Mac Lane 00] S. Mac Lane (2000) Categories for the Working Mathematician (2nd edition), SpringerVerlag.

[Penrose 71] R. Penrose (1971) Applications of negative dimensional tensors. In: Combinatorial Mathematics and its Applications, D. Welsh (Ed), pages 221-244. Academic Press.

[Perdrix 05] S. Perdrix (2005) State transfer instead of teleportation in measurement-based quantum computation. International Journal of Quantum Information 3, 219-223. arXiv:quant-ph/0402204

[Selinger 07] P. Selinger (2007) Dagger compact closed categories and completely positive maps. Electronic Notes in Theoretical Computer Science 170, 139-163.

[Selinger 11a] P. Selinger (2011) A survey of graphical languages for monoidal categories. In: New Structures for Physics, B. Coecke (ed), 275-337, Springer-Verlag. arXiv:0908.3347

[Selinger 11b] P. Selinger (2011) Finite dimensional Hilbert spaces are complete for dagger compact closed categories. Electronic Notes in Theoretical Computer Science 270 (1), 113-119. 
[Selinger 10] P. Selinger (2010) Autonomous categories in which $A$ is isomorphic to $A$ *. In: Proceedings of the 7th International Workshop on Quantum Physics and Logic (QPL 2010), Oxford.

[Wootters and Zurek 82] W. K. Wootters and W. Zurek (1982) A single quantum cannot be cloned. Nature 299, 802-803.

[Zurek 91] W. H. Zurek (1991) Decoherence and the Transition from Quantum to Classical. Physics Today 44, 36-44. arXiv:quant-ph/0306072 